\title{
Existence of homoclinic orbits for a class of $p$-Laplacian systems in a weighted Sobolev space
}

\section{Xiubo Shi', Qiongfen Zhang ${ }^{1 *}$ and Qi-Ming Zhang ${ }^{2}$}

\section{"Correspondence:}

qfzhangcsu@163.com

${ }^{1}$ College of Science, Guilin

University of Technology, Guilin,

Guangxi 541004, P.R. China

Full list of author information is

available at the end of the article

\section{Abstract}

By applying the mountain pass theorem and symmetric mountain pass theorem in critical point theory, the existence of at least one or infinitely many homoclinic solutions is obtained for the following $p$-Laplacian system:

$$
\frac{d}{d t}\left(|\dot{u}(t)|^{p-2} \dot{u}(t)\right)-a(t)|u(t)|^{q-p} u(t)+\nabla W(t, u(t))=0,
$$

where $1<p<(q+2) / 2, q>2, t \in \mathbb{R}, u \in \mathbb{R}^{N}, a \in C(\mathbb{R}, \mathbb{R})$ and $W \in C^{1}\left(\mathbb{R} \times \mathbb{R}^{N}, \mathbb{R}\right)$ are not periodic in $t$.

MSC: $34 \mathrm{C} 37 ; 35 \mathrm{~A} 15 ; 37 J 45 ; 47 J 30$

Keywords: homoclinic solutions; variational methods; weighted $L^{q-p+2}$ space; p-Laplacian systems

\section{Introduction}

Consider homoclinic solutions of the following $p$-Laplacian system:

$$
\frac{d}{d t}\left(|\dot{u}(t)|^{p-2} \dot{u}(t)\right)-a(t)|u(t)|^{q-p} u(t)+\nabla W(t, u(t))=0, \quad t \in \mathbb{R}
$$

where $1<p<(q+2) / 2, q>2, t \in \mathbb{R}, u \in \mathbb{R}^{N}, a: \mathbb{R} \rightarrow \mathbb{R}, W: \mathbb{R} \times \mathbb{R}^{N} \rightarrow \mathbb{R}$. As usual, we say that a solution $u$ of (1.1) is a nontrivial homoclinic (to 0$)$ if $u \in C^{2}\left(\mathbb{R}, \mathbb{R}^{N}\right)$ such that $u \neq 0$, $u(t) \rightarrow 0$ as $t \rightarrow \pm \infty$.

When $p=2$, (1.1) reduces to the following second-order Hamiltonian system:

$$
\ddot{u}(t)-a(t)|u(t)|^{q-2} u(t)+\nabla W(t, u(t))=0, \quad t \in \mathbb{R} .
$$

If we take $p=2$ and $q=2$, then (1.2) reduces to the following second-order Hamiltonian system:

$$
\ddot{u}(t)-a(t) u(t)+\nabla W(t, u(t))=0, \quad t \in \mathbb{R} .
$$

The existence of homoclinic orbits for Hamiltonian systems is a classical problem and its importance in the study of the behavior of dynamical systems has been recognized by

\section{Springer}

C 2013 Shi et al.; licensee Springer. This is an Open Access article distributed under the terms of the Creative Commons Attribution License (http://creativecommons.org/licenses/by/2.0), which permits unrestricted use, distribution, and reproduction in any medium, provided the original work is properly cited. 
Poincaré [1]. Up to the year of 1990, a few of isolated results can be found, and the only method for dealing with such a problem was the small perturbation technique of Melnikov.

Recently, the existence and multiplicity of homoclinic solutions and periodic solutions for Hamiltonian systems have been extensively studied by critical point theory. For example, see [2-20] and references therein. However, few results [21,22] have been obtained in the literature for system (1.2). In [22], by introducing a suitable Sobolev space, Salvatore established the following existence results for system (1.2) when $q>2$.

Theorem A [22] Assume that $a$ and W satisfy the following conditions:

(A) Let $q>2, a(t)$ is a continuous, positive function on $\mathbb{R}$ such that for all $t \in \mathbb{R}$

$$
a(t) \geq \alpha_{0}|t|^{\beta_{0}}, \quad \alpha_{0}>0, \beta_{0}>(q-2) / 2 .
$$

(W1) $W \in C^{1}\left(\mathbb{R} \times \mathbb{R}^{N}, \mathbb{R}\right)$ and there exists a constant $\mu>q$ such that

$$
0<\mu W(t, x) \leq(\nabla W(t, x), x), \quad \forall(t, x) \in \mathbb{R} \times \mathbb{R}^{N} \backslash\{0\}
$$

(W2) $|\nabla W(t, x)|=o\left(|x|^{q-1}\right)$ as $|x| \rightarrow 0$ uniformly with respect to $t \in \mathbb{R}$.

(W3) There exists $\bar{W} \in C\left(\mathbb{R}^{N}, \mathbb{R}\right)$ such that

$$
|W(t, x)|+|\nabla W(t, x)| \leq|W(x)|, \quad \forall(t, x) \in \mathbb{R} \times \mathbb{R}^{N} .
$$

Then problem (1.2) has one nontrivial homoclinic solution.

When $W(t, x)$ is an even function in $x$, Salvatore [22] obtained the following existence theorem of an unbounded sequence of homoclinic orbits for problem (1.2) by the symmetric mountain pass theorem.

Theorem B [22] Assume that a and W satisfy (A), (W1)-(W3) and the following condition:

(W4) $W(t,-x)=W(t, x), \forall(t, x) \in \mathbb{R} \times \mathbb{R}^{N}$.

Then problem (1.2) has an unbounded sequence of homoclinic solutions.

In [21], Chen and Tang improved Theorem A and Theorem B by relaxing conditions (W1) and (W2) and removing condition (W3). Motivated mainly by the ideas of [18, 2123], we will consider homoclinic solutions of (1.1) by the mountain pass theorem and symmetric mountain pass theorem. Precisely, we obtain the following main results.

Theorem 1.1 Suppose that $a$ and $W$ satisfy the following conditions:

(A) $\quad$ Let $1<p<(q+2) / 2$ and $q>2, a(t)$ is a continuous, positive function on $\mathbb{R}$ such that for all $t \in \mathbb{R}$

$$
a(t) \geq \alpha|t|^{\beta}, \quad \alpha>0, \beta>(q-2 p+2) / p .
$$


(W5) $W(t, x)=W_{1}(t, x)-W_{2}(t, x), W_{1}, W_{2} \in C^{1}\left(\mathbb{R} \times \mathbb{R}^{N}, \mathbb{R}\right)$, and there exists a constant $R>0$ such that

$$
\frac{1}{a(t)}|\nabla W(t, x)|=o\left(|x|^{q-p+1}\right) \quad \text { as } x \rightarrow 0
$$

uniformly in $t \in(-\infty,-R] \cup[R,+\infty)$.

(W6) There is a constant $\mu>q-p+2$ such that

$$
0<\mu W_{1}(t, x) \leq\left(\nabla W_{1}(t, x), x\right), \quad \forall(t, x) \in \mathbb{R} \times \mathbb{R}^{N} \backslash\{0\} .
$$

(W7) $W_{2}(t, 0)=0$ and there exists a constant $\varrho \in(q-p+2, \mu)$ such that

$$
W_{2}(t, x) \geq 0, \quad\left(\nabla W_{2}(t, x), x\right) \leq \varrho W_{2}(t, x), \quad \forall(t, x) \in \mathbb{R} \times \mathbb{R}^{N}
$$

Then problem (1.1) has one nontrivial homoclinic solution.

Theorem 1.2 Suppose that $a$ and $W$ satisfy (A)', (W6) and the following conditions:

$(\mathrm{W} 5)^{\prime} \quad W(t, x)=W_{1}(t, x)-W_{2}(t, x), W_{1}, W_{2} \in C^{1}\left(\mathbb{R} \times \mathbb{R}^{N}, \mathbb{R}\right)$, and

$$
\frac{1}{a(t)}|\nabla W(t, x)|=o\left(|x|^{q-p+1}\right) \quad \text { as } x \rightarrow 0
$$

uniformly in $t \in \mathbb{R}$.

(W7) $W_{2}(t, 0)=0$ and there exists a constant $\varrho \in(q-p+2, \mu)$ such that

$$
\left(\nabla W_{2}(t, x), x\right) \leq \varrho W_{2}(t, x), \quad \forall(t, x) \in \mathbb{R} \times \mathbb{R}^{N} .
$$

Then problem (1.1) has one nontrivial homoclinic solution.

Theorem 1.3 Suppose that $a$ and W satisfy (A)' and (W4)-(W7). Then problem (1.1) has an unbounded sequence of homoclinic solutions.

Theorem 1.4 Suppose that $a$ and W satisfy (A)', (W4), (W5)', (W6), (W7)'. Then problem (1.1) has an unbounded sequence of homoclinic solutions.

Remark 1.1 When $p=2$, condition (A)' reduces to condition (A). Obviously, Theorem 1.1Theorem 1.4 generalize and improve Theorem A, Theorem B and the corresponding results in [21]. It is easy to see that our results hold true even if $p=2$. To the best of our knowledge, similar results for problem (1.1) cannot be seen in the literature; from this point, our results are new.

Remark 1.2 If $p=2$ and $q=2$, then problem (1.1) reduces to problem (1.3). As pointed out in [23], Theorem A can be proved by replacing (A) with the more general assumption: $a(t) \rightarrow+\infty$ as $|t| \rightarrow+\infty$.

The rest of this paper is organized as follows. In Section 2, some preliminaries are presented and we establish an embedding result. In Section 3, we give the proofs of our results. In Section 4, some examples are given to illustrate our results. 


\section{Preliminaries}

We set, for any real number $1 \leq h<+\infty$,

$$
L^{h}=L^{h}\left(\mathbb{R}, \mathbb{R}^{N}\right), \quad L^{\infty}=L^{\infty}\left(\mathbb{R}, \mathbb{R}^{N}\right)
$$

with the usual norms

$$
\|u\|_{h}=\left(\int_{\mathbb{R}}|u(t)|^{h} d t\right)^{1 / h}, \quad\|u\|_{\infty}=\max _{t \in \mathbb{R}}|u(t)|
$$

Let

$$
W^{1, p}=W^{1, p}\left(\mathbb{R}, \mathbb{R}^{N}\right)=\left\{u: \mathbb{R} \rightarrow \mathbb{R}^{N} \mid u \text { is absolutely continuous, } u, \dot{u} \in L^{p}\left(\mathbb{R}, \mathbb{R}^{N}\right)\right\}
$$

be the Sobolev space with the norm given by

$$
\|u\|_{W^{1, p}}=\left(\int_{\mathbb{R}}\left[|\dot{u}(t)|^{p}+|u(t)|^{p}\right] d t\right)^{1 / p} .
$$

If $\sigma$ is a positive, continuous function on $\mathbb{R}$ and $1<s<+\infty$, let

$$
L_{\sigma}^{s}=L_{\sigma}^{s}\left(\mathbb{R}, \mathbb{R}^{N} ; \sigma\right)=\left\{\left.u \in L_{\mathrm{loc}}^{1}\left(\mathbb{R}, \mathbb{R}^{N}\right)\left|\int_{\mathbb{R}} \sigma(t)\right| u(t)\right|^{s} d t<+\infty\right\} .
$$

$L_{\sigma}^{s}$ equipped with the norm

$$
\|u\|_{s, \sigma}=\left(\int_{\mathbb{R}} \sigma(t)|u(t)|^{s} d t\right)^{1 / s}
$$

is a reflexive Banach space. When $s=+\infty$, we set

$$
L_{\sigma}^{\infty}=L_{\sigma}^{\infty}\left(\mathbb{R}, \mathbb{R}^{N} ; \sigma\right)=\left\{u\left|\max _{t \in \mathbb{R}} \sigma(t)\right| u(t) \mid<+\infty\right\}
$$

with the norm given by

$$
\|u\|_{\infty, \sigma}=\max _{t \in \mathbb{R}} \sigma(t)|u(t)| .
$$

Set $E=W^{1, p} \cap L_{a}^{q-p+2}$, where $a$ is the function given in condition (A)'. Then $E$ with its standard norm $\|\cdot\|$ is a reflexive Banach space. The functional $\varphi$ corresponding to (1.1) on $E$ is given by

$$
\varphi(u)=\int_{\mathbb{R}}\left[\frac{1}{p}|\dot{u}(t)|^{p}+\frac{a(t)}{q-p+2}|u(t)|^{q-p+2}-W(t, u(t))\right] d t, \quad u \in E .
$$

Clearly, it follows from (W5) or (W5)' that $\varphi: E \rightarrow \mathbb{R}$. By Theorem 2.1 of [24], we can deduce that the map

$$
u \rightarrow a(t)|u(t)|^{q-p} u(t)
$$


is continuous from $L_{a}^{q-p+2}$ in the dual space $L_{a^{-1 /(q-p+1)}}^{p_{1}}$, where $p_{1}=\frac{q-p+2}{q-p+1}$. As the embeddings $E \subset W^{1, p} \subset L^{\gamma}$ for all $\gamma \geq p$ are continuous, if (A)' and (W5) or (W5)' hold, then $\varphi \in$ $C^{1}(E, \mathbb{R})$ and one can easily check that

$$
\begin{aligned}
\left\langle\varphi^{\prime}(u), v\right\rangle= & \int_{\mathbb{R}}\left[|\dot{u}(t)|^{p-2}(\dot{u}(t), \dot{v}(t))+a(t)|u(t)|^{q-p}(u(t), v(t))\right. \\
& -(\nabla W(t, u(t)), v(t))] d t, \quad u \in E .
\end{aligned}
$$

Furthermore, the critical points of $\varphi$ in $E$ are classical solutions of (1.1) with $u( \pm \infty)=0$.

To prove our results, we need the following generalization of the Lebesgue dominated convergence theorem.

Lemma 2.1 [25] Let $\left\{f_{n}(t)\right\}$ and $\left\{g_{n}(t)\right\}$ be two sequences of measurable functions on a measurable set $A$, and let

$$
\left|f_{n}(t)\right| \leq g_{n}(t) \text { for a.e. } t \in A \text {. }
$$

If

$$
\lim _{n \rightarrow \infty} f_{n}(t)=f(t), \quad \lim _{n \rightarrow \infty} g_{n}(t)=g(t) \quad \text { for a.e. } t \in A
$$

and

$$
\lim _{n \rightarrow \infty} \int_{A} g_{n}(t) d t=\int_{A} g(t) d t<+\infty
$$

then

$$
\lim _{n \rightarrow \infty} \int_{A} f_{n}(t) d t=\int_{A} f(t) d t .
$$

The following lemma is an improvement result of [23] in which the author considered the case $p=2$.

Lemma 2.2 If a satisfies assumption (A)', then

the embedding $L_{a}^{q-p+2} \subset L^{p}$ is continuous.

Moreover, there exists a Sobolev space Z such that

the embeddings $L_{a}^{q-p+2} \subset Z \subset L^{p}$ are continuous,

the embedding $W^{1, p} \cap Z \subset L^{p}$ is compact.

Proof Let $\theta=(q-p+2) /(q-2 p+2), \theta^{\prime}=(q-p+2) / p$, we have

$$
\begin{aligned}
\|u\|_{p}^{p} & =\int_{\mathbb{R}} a^{-1 / \theta^{\prime}} a^{1 / \theta^{\prime}}|u|^{p} d t \\
& \leq\left(\int_{\mathbb{R}} a^{-\theta / \theta^{\prime}} d t\right)^{1 / \theta}\left(\int_{\mathbb{R}} a|u|^{p \theta^{\prime}} d t\right)^{1 / \theta^{\prime}}
\end{aligned}
$$




$$
\begin{aligned}
& =a_{1}\left(\int_{\mathbb{R}} a|u|^{q-p+2} d t\right)^{p / q-p+2} \\
& =a_{1}\|u\|_{q-p+2, a}^{p}
\end{aligned}
$$

where from $(\mathrm{A})^{\prime}, a_{1}=\left(\int_{\mathbb{R}} a^{-p /(q-2 p+2)} d t\right)^{(q-2 p+2) /(q-p+2)}<+\infty$. Then (2.3) holds.

By (A)', there exists a continuous positive function $\rho$ such that $\rho(t) \rightarrow+\infty$ as $|t| \rightarrow+\infty$ and

$$
a_{2}=\left(\int_{\mathbb{R}} \rho^{\theta} a^{-\theta / \theta^{\prime}} d t\right)^{1 / \theta}<+\infty
$$

Since

$$
\begin{aligned}
\|u\|_{p, \rho}^{p} & =\int_{\mathbb{R}} \rho|u|^{p} d t=\int_{\mathbb{R}} \rho a^{-1 / \theta^{\prime}} a^{1 / \theta^{\prime}}|u|^{p} d t \\
& \leq\left(\int_{\mathbb{R}} \rho^{\theta} a^{-\theta / \theta^{\prime}} d t\right)^{1 / \theta}\left(\int_{\mathbb{R}} a|u|^{q-p+2} d t\right)^{1 / \theta^{\prime}} \\
& =a_{2}\|u\|_{q-p+2, a}^{p}
\end{aligned}
$$

(2.4) holds by taking $Z=L_{\rho}^{p}$.

Finally, as $W^{1, p} \cap Z$ is the weighted Sobolev space $\Gamma^{1, p}(\mathbb{R}, \rho, 1)$, it follows from [24] that (2.5) holds.

The following two lemmas are the mountain pass theorem and symmetric mountain pass theorem, which are useful in the proofs of our theorems.

Lemma 2.3 [26] Let E be a real Banach space and $I \in C^{1}(E, \mathbb{R})$ satisfying $(P S)$-condition. Suppose $I(0)=0$ and:

(i) There exist constants $\rho, \alpha>0$ such that $I_{\partial B_{\rho}(0)} \geq \alpha$.

(ii) There exists an $e \in E \backslash \bar{B}_{\rho}(0)$ such that $I(e) \leq 0$.

Then I possesses a critical value $c \geq \alpha$ which can be characterized as

$$
c=\inf _{h \in \Phi} \max _{s \in[0,1]} I(h(s))
$$

where $\Phi=\{h \in C([0,1], E) \mid h(0)=0, h(1)=e\}$ and $B_{\rho}(0)$ is an open ball in $E$ of radius $\rho$ centered at 0 .

Lemma 2.4 [26] Let $E$ be a real Banach space and $I \in C^{1}(E, \mathbb{R})$ with I even. Assume that $I(0)=0$ and I satisfies (PS)-condition, (i) of Lemma 2.3 and the following condition:

(iii) For each finite dimensional subspace $E^{\prime} \subset E$, there is $r=r\left(E^{\prime}\right)>0$ such that $I(u) \leq 0$ for $u \in E^{\prime} \backslash B_{r}(0), B_{r}(0)$ is an open ball in $E$ of radius $r$ centered at 0 .

Then I possesses an unbounded sequence of critical values.

Lemma 2.5 Assume that (W6) and (W7) or (W7)' hold. Then, for every $(t, x) \in \mathbb{R} \times \mathbb{R}^{N}$,

(i) $s^{-\mu} W_{1}(t, s x)$ is nondecreasing on $(0,+\infty)$;

(ii) $s^{-\varrho} W_{2}(t, s x)$ is nonincreasing on $(0,+\infty)$.

The proof of Lemma 2.5 is routine and we omit it. 


\section{Proofs of theorems}

Proof of Theorem 1.1 Step 1. The functional $\varphi$ satisfies (PS)-condition. Let $\left\{u_{n}\right\} \subset E$ satisfying $\varphi\left(u_{n}\right)$ be bounded and $\varphi^{\prime}\left(u_{n}\right) \rightarrow 0$ as $n \rightarrow \infty$. Hence, there exists a constant $C_{1}>0$ such that

$$
\left|\varphi\left(u_{n}\right)\right| \leq C_{1}, \quad\left\|\varphi^{\prime}\left(u_{n}\right)\right\|_{E^{*}} \leq \mu C_{1} .
$$

It is well known [27] that there exists a constant $C_{2}>0$ such that

$$
\|u\|_{\infty} \leq C_{2}\|u\|, \quad u \in E .
$$

From (2.1), (2.2), (3.1), (W6) and (W7), we have

$$
\begin{aligned}
p C_{1}+p C_{1}\left\|u_{n}\right\| \geq & p \varphi\left(u_{n}\right)-\frac{p}{\mu}\left\langle\varphi^{\prime}\left(u_{n}\right), u_{n}\right\rangle \\
= & \frac{\mu-p}{\mu}\left\|\dot{u}_{n}\right\|_{p}^{p}+p \int_{\mathbb{R}}\left[W_{2}\left(t, u_{n}(t)\right)-\frac{1}{\mu}\left(\nabla W_{2}\left(t, u_{n}(t)\right), u_{n}(t)\right)\right] d t \\
& -p \int_{\mathbb{R}}\left[W_{1}\left(t, u_{n}(t)\right)-\frac{1}{\mu}\left(\nabla W_{1}\left(t, u_{n}(t)\right), u_{n}(t)\right)\right] d t \\
& +\left(\frac{p}{q-p+2}-\frac{p}{\mu}\right) \int_{\mathbb{R}} a(t)\left|u_{n}(t)\right|^{q-p+2} d t \\
\geq & \frac{\mu-p}{\mu}\left\|\dot{u}_{n}\right\|_{p}^{p}+\left(\frac{p}{q-p+2}-\frac{p}{\mu}\right)\left\|u_{n}\right\|_{q-p+2, a}^{q-p+2} .
\end{aligned}
$$

It follows from Lemma $2.2, p<(q+2) / 2, \mu>q-p+2$ and the above inequalities that there exists a constant $C_{3}>0$ such that

$$
\left\|u_{n}\right\| \leq C_{3}, \quad n \in \mathbb{N}
$$

Now we prove that $u_{n} \rightarrow u_{0}$ in $E$. Passing to a subsequence if necessary, it can be assumed that $u_{n} \rightarrow u_{0}$ in $E$. From Lemma 2.2, we have $u_{n} \rightarrow u_{0}$ in $L^{p}$. From (3.2) and (3.3), we have

$$
\left\|u_{n}\right\|_{\infty} \leq C_{2}\left\|u_{n}\right\| \leq C_{2} C_{3}, \quad u_{n} \in E .
$$

Inequality (3.4) implies that $\left|u_{n}(t)\right| \leq C_{2} C_{3}$ for all $t \in \mathbb{R}$. By (W5), we know that

$$
\frac{|\nabla W(t, x)|}{a(t)|x|^{q-p+1}} \rightarrow 0 \quad \text { as } x \rightarrow 0,
$$

which implies that for any given constant $C>0$, there exists a constant $C^{\prime}>0$ related to $C$ such that

$$
\frac{|\nabla W(t, x)|}{a(t)|x|^{q-p+1}} \leq C^{\prime} \quad \text { for }|x| \leq C .
$$

Hence, there exists a constant $C_{4}>0$ such that

$$
|\nabla W(t, x)| \leq C_{4} a(t)|x|^{q-p+1} \quad \text { for }|x| \leq C_{2} C_{3} .
$$


Hence, from (3.5), we have

$$
\begin{aligned}
& \left|\nabla W\left(t, u_{n}(t)\right)-\nabla W\left(t, u_{0}(t)\right)\right|^{p^{\prime}} \\
& \quad \leq\left[C_{4} a(t)\left(\left|u_{n}(t)\right|^{q-p+1}+\left|u_{0}(t)\right|^{q-p+1}\right)\right]^{p^{\prime}} \\
& \quad \leq\left[C_{4} 2^{q-p+1} a(t)\left|u_{n}(t)-u_{0}(t)\right|^{q-p+1}+C_{4}\left(1+2^{q-p+1}\right) a(t)\left|u_{0}(t)\right|^{q-p+1}\right]^{p^{\prime}} \\
& \quad \leq 2^{p^{\prime}(q-p+2)} C_{4}^{p^{\prime}} a^{p^{\prime}}(t)\left|u_{n}(t)-u_{0}(t)\right|^{p^{\prime}(q-p+1)}+2^{p^{\prime}} C_{4}^{p^{\prime}}\left(1+2^{q-p+1}\right)^{p^{\prime}} a^{p^{\prime}}(t)\left|u_{0}(t)\right|^{p^{\prime}(q-p+1)} \\
& \quad:=g_{n}(t),
\end{aligned}
$$

where $p^{\prime}=\frac{p}{p-1}$. Moreover, since $a(t)$ is a positive continuous function on $\mathbb{R}, p<q-p+2$ and $u_{n}(t) \rightarrow u_{0}(t)$ for almost every $t \in \mathbb{R}$, we have

$$
\lim _{n \rightarrow \infty} g_{n}(t)=2^{p^{\prime}} C_{4}^{p^{\prime}}\left(1+2^{q-p+1}\right)^{p^{\prime}} a^{p^{\prime}}(t)\left|u_{0}(t)\right|^{p(q-p+1)}:=g(t) \quad \text { for a.e. } t \in \mathbb{R}
$$

and

$$
\begin{aligned}
\lim _{n \rightarrow \infty} \int_{\mathbb{R}} g_{n}(t) d t= & \lim _{n \rightarrow \infty} \int_{\mathbb{R}}\left[2^{p^{\prime}(q-p+2)} C_{4}^{p^{\prime}} a^{p^{\prime}}(t)\left|u_{n}(t)-u_{0}(t)\right|^{p^{\prime}(q-p+1)}\right. \\
& \left.+2^{p^{\prime}} C_{4}^{p^{\prime}}\left(1+2^{q-p+1}\right)^{p^{\prime}} a^{p^{\prime}}(t)\left|u_{0}(t)\right|^{p^{\prime}(q-p+1)}\right] d t \\
= & 2^{p^{\prime}(q-p+2)} C_{4}^{p^{\prime}} \lim _{n \rightarrow \infty} \int_{\mathbb{R}} a^{p^{\prime}}(t)\left|u_{n}(t)-u_{0}(t)\right|^{p^{\prime}(q-p+1)} d t \\
& +2^{p^{\prime}} C_{4}^{p^{\prime}}\left(1+2^{q-p+1}\right)^{p^{\prime}} \int_{\mathbb{R}} a^{p^{\prime}}(t)\left|u_{0}(t)\right|^{p^{\prime}(q-p+1)} d t \\
= & 2^{p^{\prime}} C_{4}^{p^{\prime}}\left(1+2^{q-p+1}\right)^{p^{\prime}} \int_{\mathbb{R}} a^{p^{\prime}}(t)\left|u_{0}(t)\right|^{p^{\prime}(q-p+1)} d t \\
= & \int_{\mathbb{R}} g(t) d t<+\infty .
\end{aligned}
$$

It follows from Lemma 2.1, (3.6) and the above inequalities that

$$
\lim _{n \rightarrow \infty} \int_{\mathbb{R}}\left|\nabla W\left(t, u_{n}(t)\right)-\nabla W\left(t, u_{0}(t)\right)\right|^{p^{\prime}} d t=0
$$

This shows that

$$
\nabla W\left(t, u_{n}\right) \rightarrow \nabla W\left(t, u_{0}\right) \quad \text { in } L^{p^{\prime}}\left(\mathbb{R}, \mathbb{R}^{N}\right)
$$

From (2.2), we have

$$
\begin{aligned}
& \left.\left\langle\varphi^{\prime}\left(u_{n}\right)-\varphi^{\prime}\left(u_{0}\right), u_{n}-u_{0}\right)\right\rangle \\
& =\int_{\mathbb{R}}\left(\left|\dot{u}_{n}(t)\right|^{p-2} \dot{u}_{n}(t)-\left|\dot{u}_{0}(t)\right|^{p-2} \dot{u}_{0}(t), \dot{u}_{n}(t)-\dot{u}_{0}(t)\right) d t \\
& \quad+\int_{\mathbb{R}} a(t)\left(\left|u_{n}(t)\right|^{q-p} u_{n}(t)-\left|u_{0}(t)\right|^{q-p} u_{0}(t)\right)\left(u_{n}(t)-u_{0}(t)\right) d t \\
& \quad-\int_{\mathbb{R}}\left(\nabla W\left(t, u_{n}(t)\right)-\nabla W\left(t, u_{0}(t)\right), u_{n}(t)-u_{0}(t)\right) d t
\end{aligned}
$$




$$
\begin{aligned}
& \geq\left\|\dot{u}_{n}\right\|_{p}^{p}+\left\|\dot{u}_{0}\right\|_{p}^{p}-\left\|\dot{u}_{0}\right\|_{p}\left\|\dot{u}_{n}\right\|_{p}^{p-1}-\left\|\dot{u}_{n}\right\|_{p}\left\|\dot{u}_{0}\right\|_{p}^{p-1} \\
& \quad+\int_{\mathbb{R}} a(t)\left(\left|u_{n}(t)\right|^{q-p} u_{n}(t)-\left|u_{0}(t)\right|^{q-p} u_{0}(t)\right)\left(u_{n}(t)-u_{0}(t)\right) d t \\
& \quad-\int_{\mathbb{R}}\left(\nabla W\left(t, u_{n}(t)\right)-\nabla W\left(t, u_{0}(t)\right), u_{n}(t)-u_{0}(t)\right) d t \\
& =\left(\left\|\dot{u}_{n}\right\|_{p}^{p-1}-\left\|\dot{u}_{0}\right\|_{p}^{p-1}\right)\left(\left\|\dot{u}_{n}\right\|_{p}-\left\|\dot{u}_{0}\right\|_{p}\right) \\
& \quad+\int_{\mathbb{R}} a(t)\left(\left|u_{n}(t)\right|^{q-p} u_{n}(t)-\left|u_{0}(t)\right|^{q-p} u_{0}(t)\right)\left(u_{n}(t)-u_{0}(t)\right) d t \\
& \quad-\int_{\mathbb{R}}\left(\nabla W\left(t, u_{n}(t)\right)-\nabla W\left(t, u_{0}(t)\right), u_{n}(t)-u_{0}(t)\right) d t .
\end{aligned}
$$

It is easy to see that for any $\alpha>1$ there exists a constant $C_{5}>0$ such that

$$
\left(|x|^{\alpha-1} x-|y|^{\alpha-1} y\right)(x-y) \geq C_{5}|x-y|^{\alpha+1}, \quad \forall x, y \in \mathbb{R}
$$

Inequality (3.9) implies that

$$
\left(\left\|\dot{u}_{n}\right\|_{p}^{p-1}-\left\|\dot{u}_{0}\right\|_{p}^{p-1}\right)\left(\left\|\dot{u}_{n}\right\|_{p}-\left\|\dot{u}_{0}\right\|_{p}\right) \geq C_{5}^{\prime}\left|\left\|\dot{u}_{n}\right\|_{p}-\left\|\dot{u}_{0}\right\|_{p}\right|^{p}
$$

and

$$
\begin{aligned}
& \int_{\mathbb{R}} a(t)\left(\left|u_{n}(t)\right|^{q-p} u_{n}(t)-\left|u_{0}(t)\right|^{q-p} u_{0}(t)\right)\left(u_{n}(t)-u_{0}(t)\right) d t \\
& \quad \geq C_{5}^{\prime \prime} \int_{\mathbb{R}} a(t)\left|u_{n}(t)-u_{0}(t)\right|^{q-p+2} d t
\end{aligned}
$$

where $C_{5}^{\prime}$ and $C_{5}^{\prime \prime}$ are positive constants. Since $\varphi^{\prime}\left(u_{n}\right) \rightarrow 0$ as $n \rightarrow+\infty, u_{n} \rightarrow u$ in $E$ and the embeddings $E \subset W^{1, p} \subset L^{\gamma}$ for all $\gamma \geq p$ are continuous, it follows from Lemma 2.2, (3.7), (3.8), (3.10) and (3.11) that

$$
\left\|\dot{u}_{n}\right\|_{p} \rightarrow\left\|\dot{u}_{0}\right\|_{p} \quad \text { as } n \rightarrow \infty
$$

and

$$
\int_{\mathbb{R}} a(t)\left|u_{n}(t)\right|^{q-p+2} d t \rightarrow \int_{\mathbb{R}} a(t)\left|u_{0}(t)\right|^{q-p+2} d t \quad \text { as } n \rightarrow \infty
$$

Hence, by (3.12) and (3.13), $u_{n} \rightarrow u_{0}$ in $E$. This shows that $\varphi$ satisfies (PS)-condition.

Step 2. From (W5), there exists $\delta \in(0,1)$ such that

$$
|\nabla W(t, x)| \leq \frac{1}{p} a(t)|x|^{q-p+1} \quad \text { for }|t| \geq R,|x| \leq \delta
$$

By (3.14), we have

$$
|W(t, x)| \leq \frac{1}{p(q-p+2)} a(t)|x|^{q-p+2} \quad \text { for }|t| \geq R,|x| \leq \delta .
$$


Let

$$
C_{6}=\sup \left\{\frac{W_{1}(t, x)}{a(t)}|t \in[-R, R], x \in \mathbb{R},| x \mid=1\right\} .
$$

Set $\sigma=\min \left\{1 /\left(p(q-p+2) C_{6}+1\right)^{1 /(\mu-q+p-2)}, \delta\right\}$ and $\|u\|=\sigma / C_{2}:=\rho$, it follows from (3.2) that

$$
\|u\|_{\infty} \leq C_{2}\|u\| \leq \sigma,
$$

which shows that $|u(t)| \leq \sigma \leq \delta<1$. From Lemma 2.5(i) and (3.16), we have

$$
\begin{aligned}
\int_{-R}^{R} W_{1}(t, u(t)) d t & \leq \int_{\{t \in[-R, R]: u(t) \neq 0\}} W_{1}\left(t, \frac{u(t)}{|u(t)|}\right)|u(t)|^{\mu} d t \\
& \leq C_{6} \int_{-R}^{R} a(t)|u(t)|^{\mu} d t \\
& \leq C_{6} \sigma^{\mu-q+p-2} \int_{-R}^{R} a(t)|u(t)|^{q-p+2} d t \\
& \leq \frac{1}{p(q-p+2)} \int_{-R}^{R} a(t)|u(t)|^{q-p+2} d t .
\end{aligned}
$$

It follows from (W7), (3.15), (3.17) that

$$
\begin{aligned}
\varphi(u)= & \frac{1}{p} \int_{\mathbb{R}}|\dot{u}(t)|^{p} d t+\int_{\mathbb{R}} \frac{a(t)}{q-p+2}|u(t)|^{q-p+2} d t-\int_{\mathbb{R}} W(t, u(t)) d t \\
= & \frac{1}{p}\|\dot{u}\|_{p}^{p}+\frac{1}{q-p+2}\|u\|_{q-p+2, a}^{q-p+2}-\int_{\mathbb{R} \backslash[-R, R]} W(t, u(t)) d t-\int_{-R}^{R} W(t, u(t)) d t \\
\geq & \frac{1}{p}\|\dot{u}\|_{p}^{p}+\frac{1}{q-p+2}\|u\|_{q-p+2, a}^{q-p+2}-\int_{-R}^{R} W_{1}(t, u(t)) d t \\
& -\int_{\mathbb{R} \backslash[-R, R]} \frac{1}{p(q-p+2)} a(t)|u(t)|^{q-p+2} d t \\
\geq & \frac{1}{p}\|\dot{u}\|_{p}^{p}+\frac{1}{q-p+2}\|u\|_{q-p+2, a}^{q-p+2}-\frac{1}{p(q-p+2)} \int_{-R}^{R} a(t)|u(t)|^{q-p+2} d t \\
& -\int_{\mathbb{R} \backslash[-R, R]} \frac{1}{p(q-p+2)} a(t)|u(t)|^{q-p+2} d t \\
= & \frac{1}{p}\|\dot{u}\|_{p}^{p}+\frac{p-1}{p(q-p+2)}\|u\|_{q-p+2, a}^{q-p+2} .
\end{aligned}
$$

Therefore, we can choose a constant $\alpha>0$ depending on $\rho$ such that $\varphi(u) \geq \alpha$ for any $u \in E$ with $\|u\|=\rho$.

Step 3. From Lemma 2.5(ii) and (3.2), we have for any $u \in E$

$$
\begin{aligned}
\int_{-2}^{2} W_{2}(t, u(t)) d t & =\int_{\{t \in[-2,2]:|u(t)|>1\}} W_{2}(t, u(t)) d t+\int_{\{t \in[-2,2]:|u(t)| \leq 1\}} W_{2}(t, u(t)) d t \\
& \leq \int_{\{t \in[-2,2]:|u(t)|>1\}} W_{2}\left(t, \frac{u(t)}{|u(t)|}\right)|u(t)|^{\varrho} d t+\int_{-2}^{2} \max _{|x| \leq 1} W_{2}(t, x) d t
\end{aligned}
$$




$$
\begin{aligned}
& \leq\|u\|_{\infty}^{\varrho} \int_{-2}^{2} \max _{|x|=1} W_{2}(t, x) d t+\int_{-2}^{2} \max _{|x| \leq 1} W_{2}(t, x) d t \\
& \leq C_{2}^{\varrho}\|u\|^{\varrho} \int_{-2}^{2} \max _{|x|=1} W_{2}(t, x) d t+\int_{-2}^{2} \max _{|x| \leq 1} W_{2}(t, x) d t \\
& =C_{7}\|u\|^{\varrho}+C_{8},
\end{aligned}
$$

where $C_{7}=C_{2}^{\varrho} \int_{-2}^{2} \max _{|x|=1} W_{2}(t, x) d t, C_{8}=\int_{-2}^{2} \max _{|x| \leq 1} W_{2}(t, x) d t$. Take $\omega \in E$ such that

$$
|\omega(t)|= \begin{cases}1 & \text { for }|t| \leq 1 \\ 0 & \text { for }|t| \geq 2\end{cases}
$$

and $|\omega(t)| \leq 1$ for $|t| \in(1,2]$. For $s>1$, from Lemma 2.5(i) and (3.19), we get

$$
\int_{-1}^{1} W_{1}(t, s \omega(t)) d t \geq s^{\mu} \int_{-1}^{1} W_{1}(t, \omega(t)) d t=C_{9} s^{\mu}
$$

where $C_{9}=\int_{-1}^{1} W_{1}(t, \omega(t)) d t>0$. From (W7), (2.1), (3.18), (3.19), (3.20), we get for $s>1$

$$
\begin{aligned}
\varphi(s \omega) & =\frac{s^{p}}{p}\|\dot{\omega}\|_{p}^{p}+\frac{s^{q-p+2}}{q-p+2}\|\omega\|_{q-p+2, a}^{q-p+2}+\int_{\mathbb{R}}\left[W_{2}(t, s \omega(t))-W_{1}(t, s \omega(t))\right] d t \\
& \leq \frac{s^{p}}{p}\|\dot{\omega}\|_{p}^{p}+\frac{s^{q-p+2}}{q-p+2}\|\omega\|_{q-p+2, a}^{q-p+2}+\int_{-2}^{2} W_{2}(t, s \omega(t)) d t-\int_{-1}^{1} W_{1}(t, s \omega(t)) d t \\
& \leq \frac{s^{p}}{p}\|\dot{\omega}\|_{p}^{p}+\frac{s^{q-p+2}}{q-p+2}\|\omega\|_{q-p+2, a}^{q-p+2}+C_{7} s^{\varrho}\|\omega\|^{\varrho}+C_{8}-C_{9} s^{\mu}
\end{aligned}
$$

Since $\mu>\varrho>q-p+2$ and $C_{9}>0$, it follows from (3.21) that there exists $s_{1}>1$ such that $\left\|s_{1} \omega\right\|>\rho$ and $\varphi\left(s_{1} \omega\right)<0$. Let $e=s_{1} \omega(t)$, then $e \in E,\|e\|=\left\|s_{1} \omega\right\|>\rho$ and $\varphi(e)=\varphi\left(s_{1} \omega\right)<0$. By Lemma 2.3, $\varphi$ has a critical value $d>\alpha$ given by

$$
d=\inf _{g \in \Phi} \max _{s \in[0,1]} \varphi(g(s))
$$

where

$$
\Phi=\{g \in C([0,1], E): g(0)=0, g(1)=e\} .
$$

Hence, there exists $u^{*} \in E$ such that

$$
\varphi\left(u^{*}\right)=d, \quad \varphi^{\prime}\left(u^{*}\right)=0 .
$$

The function $u^{*}$ is a desired solution of problem (1.1). Since $d>0, u^{*}$ is a nontrivial homoclinic solution. The proof is complete.

Proof of Theorem 1.2 In the proof of Theorem 1.1, the condition $W_{2}(t, x) \geq 0$ in (W7) is only used in the proofs of (3.3) and Step 2. Therefore, we only need to prove that (3.3) and Step 2 still hold if we use (W5)' and (W7)' instead of (W5) and (W7). We first prove that 
(3.3) holds. From (W6), (W7)', (2.1), (2.2) and (3.1), we have

$$
\begin{aligned}
p(q-p+2) C_{1}+\frac{p(q-p+2) C_{1} \mu}{\varrho}\left\|u_{n}\right\| \\
\geq p(q-p+2) \varphi\left(u_{n}\right)-\frac{p(q-p+2)}{\varrho}\left\langle\varphi^{\prime}\left(u_{n}\right), u_{n}\right\rangle \\
=\frac{(\varrho-p)(q-p+2)}{\varrho}\left\|\dot{u}_{n}\right\|_{p}^{p} \\
\quad+p(q-p+2) \int_{\mathbb{R}}\left[W_{2}\left(t, u_{n}(t)\right)-\frac{1}{\varrho}\left(\nabla W_{2}\left(t, u_{n}(t)\right), u_{n}(t)\right)\right] d t \\
\quad-p(q-p+2) \int_{\mathbb{R}}\left[W_{1}\left(t, u_{n}(t)\right)-\frac{1}{\varrho}\left(\nabla W_{1}\left(t, u_{n}(t)\right), u_{n}(t)\right)\right] d t \\
\quad+p\left(1-\frac{q-p+2}{\varrho}\right) \int_{\mathbb{R}} a(t)\left|u_{n}(t)\right|^{q-p+2} d t \\
\geq \\
\quad \frac{(\varrho-p)(q-p+2)}{\varrho}\left\|\dot{u}_{n}\right\|_{p}^{p}+p\left(1-\frac{q-p+2}{\varrho}\right)\left\|u_{n}\right\|_{q-p+2, a}^{q-p+2},
\end{aligned}
$$

which implies that there exists a constant $C_{3}>0$ such that (3.3) holds. Next, we prove Step 2 still holds. From (W5)', there exists $\delta \in(0,1)$ such that

$$
|\nabla W(t, x)| \leq \frac{1}{p} a(t)|x|^{q-p+1} \quad \text { for } t \in \mathbb{R},|x| \leq \delta
$$

By (3.23), we have

$$
|W(t, x)| \leq \frac{1}{p(q-p+2)} a(t)|x|^{q-p+2} \quad \text { for } t \in \mathbb{R},|x| \leq \delta .
$$

Let $0<\sigma \leq \delta$ and $\|u\|=\sigma / C_{2}:=\rho$, it follows from (3.2) that

$$
\|u\|_{\infty} \leq C_{2}\|u\| \leq \sigma
$$

which shows that $|u(t)| \leq \sigma \leq \delta<1$. It follows from (2.1) and (3.24) that

$$
\begin{aligned}
\varphi(u) & =\frac{1}{p} \int_{\mathbb{R}}|\dot{u}(t)|^{p} d t+\int_{\mathbb{R}} \frac{a(t)}{q-p+2}|u(t)|^{q-p+2} d t-\int_{\mathbb{R}} W(t, u(t)) d t \\
& \geq \frac{1}{p}\|\dot{u}\|_{p}^{p}+\frac{1}{q-p+2}\|u\|_{q-p+2, a}^{q-p+2}-\int_{\mathbb{R}} \frac{1}{p(q-p+2)} a(t)|u(t)|^{q-p+2} d t \\
& =\frac{1}{p}\|\dot{u}\|_{p}^{p}+\frac{p-1}{p(q-p+2)}\|u\|_{q-p+2, a}^{q-p+2} .
\end{aligned}
$$

Therefore, we can choose a constant $\alpha>0$ depending on $\rho$ such that $\varphi(u) \geq \alpha$ for any $u \in E$ with $\|u\|=\rho$. The proof of Theorem 1.2 is complete.

Proof of Theorem 1.3 Condition (W4) shows that $\varphi$ is even. In view of the proof of Theorem 1.1, we know that $\varphi \in C^{1}(E, \mathbb{R})$ and satisfies (PS)-condition and assumptions (i) of Lemma 2.3. Now, we prove that (iii) of Lemma 2.4. Let $E^{\prime}$ be a finite dimensional subspace 
of $E$. Since all norms of a finite dimensional space are equivalent, there exists $c>0$ such that

$$
\|u\| \leq c\|u\|_{\infty} .
$$

Assume that $\operatorname{dim} E^{\prime}=m$ and $\left\{u_{1}, u_{2}, \ldots, u_{m}\right\}$ is a base of $E^{\prime}$ such that

$$
\left\|u_{i}\right\|=c, \quad i=1,2, \ldots, m .
$$

For any $u \in E^{\prime}$, there exists $\lambda_{i} \in \mathbb{R}, i=1,2, \ldots, m$ such that

$$
u(t)=\sum_{i=1}^{m} \lambda_{i} u_{i}(t) \quad \text { for } t \in \mathbb{R}
$$

Let

$$
\|u\|_{*}=\sum_{i=1}^{m}\left|\lambda_{i}\right|\left\|u_{i}\right\|
$$

It is easy to see that $\|\cdot\|_{*}$ is a norm of $E^{\prime}$. Hence, there exists a constant $c^{\prime}>0$ such that $c^{\prime}\|u\|_{*} \leq\|u\|$. Since $u_{i} \in E$, by Lemma 2.2, we can choose $R_{1}>R$ such that

$$
\left|u_{i}(t)\right|<\frac{c^{\prime} \delta}{1+c^{\prime}}, \quad|t|>R_{1}, i=1,2, \ldots, m,
$$

where $\delta$ is given in (3.24). Let

$$
\Theta=\left\{\sum_{i=1}^{m} \lambda_{i} u_{i}(t): \lambda_{i} \in \mathbb{R}, i=1,2, \ldots, m ; \sum_{i=1}^{m}\left|\lambda_{i}\right|=1\right\}=\left\{u \in E^{\prime}:\|u\|_{*}=c\right\} .
$$

Hence, for $u \in \Theta$, let $t_{0}=t_{0}(u) \in \mathbb{R}$ such that

$$
\left|u\left(t_{0}\right)\right|=\|u\|_{\infty} .
$$

Then by (3.25)-(3.28), (3.30) and (3.31), we have

$$
\begin{aligned}
c c^{\prime} & =c c^{\prime} \sum_{i=1}^{m}\left|\lambda_{i}\right|=c^{\prime} \sum_{i=1}^{m}\left|\lambda_{i}\right|\left\|u_{i}\right\|=c^{\prime}\|u\|_{*} \\
& \leq\|u\| \leq c\|u\|_{\infty}=c\left|u\left(t_{0}\right)\right| \\
& \leq c \sum_{i=1}^{m}\left|\lambda_{i}\right|\left|u_{i}\left(t_{0}\right)\right|, \quad u \in \Theta .
\end{aligned}
$$

This shows that $\left|u\left(t_{0}\right)\right| \geq c^{\prime}$ and there exists $i_{0} \in\{1,2, \ldots, m\}$ such that $\left|u_{i_{0}}\left(t_{0}\right)\right| \geq c^{\prime}$, which together with (3.29), implies that $\left|t_{0}\right| \leq R_{1}$. Let $R_{2}=R_{1}+1$ and

$$
\gamma=\min \left\{W_{1}(t, x):-R_{2} \leq t \leq R_{2}, \frac{c^{\prime}}{2^{1 / p}} \leq|x| \leq c C_{2}\right\}
$$


Since $W_{1}(t, x)>0$ for all $t \in \mathbb{R}$ and $x \in \mathbb{R}^{N} \backslash\{0\}$, and $W_{1} \in C^{1}\left(\mathbb{R} \times \mathbb{R}^{N}, \mathbb{R}\right)$, it follows that $\gamma>0$. For any $u \in E$, from Lemma 2.5(i) and (3.2), we have

$$
\begin{aligned}
\int_{-R_{2}}^{R_{2}} W_{2}(t, u(t)) d t & =\int_{\left\{t \in\left[-R_{2}, R_{2}\right]:|u(t)|>1\right\}} W_{2}(t, u(t)) d t+\int_{\left\{t \in\left[-R_{2}, R_{2}\right]:|u(t)| \leq 1\right\}} W_{2}(t, u(t)) d t \\
& \leq \int_{\left\{t \in\left[-R_{2}, R_{2}\right]:|u(t)|>1\right\}} W_{2}\left(t, \frac{u(t)}{|u(t)|}\right)|u(t)|^{\varrho} d t+\int_{-R_{2}}^{R_{2}} \max _{|x| \leq 1} W_{2}(t, x) d t \\
& \leq\|u\|_{\infty}^{\varrho} \int_{-R_{2}}^{R_{2}} \max _{|x|=1} W_{2}(t, x) d t+\int_{-R_{2}}^{R_{2}} \max _{|x| \leq 1} W_{2}(t, x) d t \\
& \leq C_{2}^{\varrho}\|u\|^{\varrho} \int_{-R_{2}}^{R_{2}} \max _{|x|=1} W_{2}(t, x) d t+\int_{-R_{2}}^{R_{2}} \max _{|x| \leq 1} W_{2}(t, x) d t \\
& =C_{10}\|u\|^{\varrho}+C_{11},
\end{aligned}
$$

where $C_{10}=C_{2}^{\varrho} \int_{-R_{2}}^{R_{2}} \max _{|x|=1} W_{2}(t, x) d t, C_{11}=\int_{-R_{2}}^{R_{2}} \max _{|x| \leq 1} W_{2}(t, x) d t$. Since $\dot{u}_{i} \in L^{p}(\mathbb{R}), i=$ $1,2, \ldots, m$, it follows that there exists $\varepsilon \in(0,1)$ such that

$$
\begin{aligned}
\int_{t+\varepsilon}^{t-\varepsilon}\left|\dot{u}_{i}(s)\right| d s & \leq(2 \varepsilon)^{1 / p^{\prime}}\left(\int_{t+\varepsilon}^{t-\varepsilon}\left|\dot{u}_{i}(s)\right|^{p} d s\right)^{1 / p} \\
& \leq(2 \varepsilon)^{1 / p^{\prime}}\left\|\dot{u}_{i}\right\|_{p} \\
& \leq \frac{c^{\prime}}{2 p} \quad \text { for } t \in \mathbb{R}, i=1,2, \ldots, m
\end{aligned}
$$

where $1 / p^{\prime}+1 / p=1$. Then, for $u \in \Theta$ with $\left|u\left(t_{0}\right)\right|=\|u\|_{\infty}$ and $t \in\left[t_{0}-\varepsilon, t_{0}+\varepsilon\right]$, it follows from (3.27), (3.30), (3.31), (3.32) and (3.35) that

$$
\begin{aligned}
|u(t)|^{p} & =\left|u\left(t_{0}\right)\right|^{p}+p \int_{t_{0}}^{t}|u(s)|^{p-2}(\dot{u}(s), u(s)) d s \\
& \geq\left|u\left(t_{0}\right)\right|^{p}-p \int_{t_{0}-\varepsilon}^{t_{0}+\varepsilon}|u(s)|^{p-1}|\dot{u}(s)| d s \\
& \geq\left|u\left(t_{0}\right)\right|^{p}-p\left|u\left(t_{0}\right)\right|^{p-1} \int_{t_{0}-\varepsilon}^{t_{0}+\varepsilon}|\dot{u}(s)| d s \\
& \geq \frac{c^{\prime}}{2}\left|u\left(t_{0}\right)\right|^{p-1} \\
& \geq \frac{c^{\prime p}}{2} .
\end{aligned}
$$

On the other hand, since $\|u\| \leq c$ for $u \in \Theta$, then

$$
|u(t)| \leq\|u\|_{\infty} \leq C_{2} c, \quad t \in \mathbb{R}, u \in \Theta
$$

Therefore, from (3.33), (3.36) and (3.37), we have

$$
\int_{-R_{2}}^{R_{2}} W_{1}(t, u(t)) d t \geq \int_{t_{0}-\varepsilon}^{t_{0}+\varepsilon} W_{1}(t, u(t)) d t \geq 2 \varepsilon \gamma \quad \text { for } u \in \Theta
$$


From (3.29) and (3.30), we have

$$
|u(t)| \leq \sum_{i=1}^{m}\left|\lambda_{i}\right|\left|u_{i}(t)\right| \leq \delta \quad \text { for }|t| \geq R_{1}, u \in \Theta .
$$

By (2.1), (3.15), (3.34), (3.38), (3.39) and Lemma 2.5, we have for $u \in \Theta$ and $r>1$

$$
\begin{aligned}
& \varphi(r u)=\frac{r^{p}}{p}\|\dot{u}\|_{p}^{p}+\frac{r^{q-p+2}}{q-p+2}\|u\|_{q-p+2, a}^{q-p+2}+\int_{\mathbb{R}}\left[W_{2}(t, r u(t))-W_{1}(t, r u(t))\right] d t \\
& \leq \frac{r^{p}}{p}\|\dot{u}\|_{p}^{p}+\frac{r^{q-p+2}}{q-p+2}\|u\|_{q-p+2, a}^{q-p+2}+r^{\varrho} \int_{\mathbb{R}} W_{2}(t, u(t)) d t-r^{\mu} \int_{\mathbb{R}} W_{1}(t, u(t)) d t \\
& =\frac{r^{p}}{p}\|\dot{u}\|_{p}^{p}+\frac{r^{q-p+2}}{q-p+2}\|u\|_{q-p+2, a}^{q-p+2}+r^{\varrho} \int_{\mathbb{R} \backslash\left(-R_{2}, R_{2}\right)} W_{2}(t, u(t)) d t \\
& -r^{\mu} \int_{\mathbb{R} \backslash\left(-R_{2}, R_{2}\right)} W_{1}(t, u(t)) d t+r^{\varrho} \int_{-R_{2}}^{R_{2}} W_{2}(t, u(t)) d t \\
& -r^{\mu} \int_{-R_{2}}^{R_{2}} W_{1}(t, u(t)) d t \\
& \leq \frac{r^{p}}{p}\|\dot{u}\|_{p}^{p}+\frac{r^{q-p+2}}{q-p+2}\|u\|_{q-p+2, a}^{q-p+2}-r^{\varrho} \int_{\mathbb{R} \backslash\left(-R_{2}, R_{2}\right)} W(t, u(t)) d t \\
& -r^{\mu} \int_{-R_{2}}^{R_{2}} W_{1}(t, u(t)) d t+r^{\varrho} \int_{-R_{2}}^{R_{2}} W_{2}(t, u(t)) d t \\
& \leq \frac{r^{p}}{p}\|\dot{u}\|_{p}^{p}+\frac{r^{q-p+2}}{q-p+2}\|u\|_{q-p+2, a}^{q-p+2}+\frac{r^{\varrho}}{p(q-p+2)} \int_{\mathbb{R} \backslash\left(-R_{2}, R_{2}\right)} a(t)|u(t)|^{q-p+2} d t \\
& +r^{\varrho}\left(C_{10}\|u\|^{\varrho}+C_{11}\right)-2 \varepsilon \gamma r^{\mu} \\
& \leq \frac{r^{p}}{p}\|\dot{u}\|_{p}^{p}+\frac{r^{q-p+2}}{q-p+2}\|u\|_{q-p+2, a}^{q-p+2}+\frac{r^{\varrho}}{p(q-p+2)}\|u\|_{q-p+2, a}^{q-p+2} \\
& +r^{\varrho}\left(C_{10}\|u\|^{\varrho}+C_{11}\right)-2 \varepsilon \gamma r^{\mu} \\
& \leq \frac{r^{p}}{p} c^{p}+\frac{r^{q-p+2}}{q-p+2} c^{q-p+2}+\frac{r^{\varrho}}{p(q-p+2)} c^{q-p+2} \\
& +C_{10}(r c)^{\varrho}+C_{11} r^{\varrho}-2 \varepsilon \gamma r^{\mu} \text {. }
\end{aligned}
$$

Since $\mu>\varrho>q-p+2>p$, we deduce that there exists $r_{0}=r_{0}\left(c, c^{\prime}, C_{10}, C_{11}, R_{1}, R_{2}, \varepsilon, \gamma\right)=$ $r_{0}\left(E^{\prime}\right)>1$ such that

$$
\varphi(r u)<0 \quad \text { for } u \in \Theta \text { and } r \geq r_{0} \text {. }
$$

It follows that

$$
\varphi(u)<0 \quad \text { for } u \in E^{\prime} \text { and }\|u\| \geq c r_{0} \text {, }
$$

which shows that (iii) of Lemma 2.4 holds. By Lemma 2.4, $\varphi$ possesses an unbounded sequence $\left\{d_{n}\right\}_{n=1}^{\infty}$ of critical values with $d_{n}=\varphi\left(u_{n}\right)$, where $u_{n}$ is such that $\varphi^{\prime}\left(u_{n}\right)=0$ for 
$n=1,2, \ldots$ If $\left\{\left\|u_{n}\right\|\right\}$ is bounded, then there exists $C_{12}>0$ such that

$$
\left\|u_{n}\right\| \leq C_{12} \quad \text { for } n \in \mathbb{N} \text {. }
$$

By (3.2) and (3.41), we get

$$
\left|u_{n}(t)\right| \leq C_{2} C_{12} \quad \text { for } n \in \mathbb{N} \text {. }
$$

From (W5), we can choose $C_{13}>0$ and $R_{3}>R$ such that

$$
|\nabla W(t, x)| \leq C_{13} a(t)|x|^{q-p+1} \quad \text { for }|t| \geq R_{3},|x| \leq C_{2} C_{12} \text {, }
$$

which implies that

$$
|W(t, x)| \leq \frac{C_{13}}{q-p+2} a(t)|x|^{q-p+2} \quad \text { for }|t| \geq R_{3},|x| \leq C_{2} C_{12}
$$

Hence, by (2.1) and (3.43), we have

$$
\begin{aligned}
\frac{1}{p} & \left\|\dot{u}_{n}\right\|_{p}^{p}+\frac{1}{q-p+2}\left\|u_{n}\right\|_{q-p+2, a}^{q-p+2} \\
& =d_{n}+\int_{\mathbb{R}} W\left(t, u_{n}(t)\right) d t \\
& =d_{n}+\int_{\mathbb{R} \backslash\left[-R_{3}, R_{3}\right]} W\left(t, u_{n}(t)\right) d t+\int_{-R_{3}}^{R_{3}} W\left(t, u_{n}(t)\right) d t \\
& \geq d_{n}-\frac{C_{13}}{q-p+2} \int_{\mathbb{R} \backslash\left[-R_{3}, R_{3}\right]} a(t)\left|u_{n}(t)\right|^{q-p+2} d t-\int_{-R_{3}}^{R_{3}}\left|W\left(t, u_{n}(t)\right)\right| d t \\
& \geq d_{n}-\frac{C_{13}}{q-p+2}\left\|u_{n}\right\|_{q-p+2, a}^{q-p+2}-\int_{-R_{3}}^{R_{3}} \max _{|x| \leq C_{2} C_{12}}|W(t, x)| d t,
\end{aligned}
$$

which, together with (3.41), implies that

$$
d_{n} \leq \frac{1}{p}\left\|\dot{u}_{n}\right\|_{p}^{p}+\frac{C_{13}+1}{q-p+2}\left\|u_{n}\right\|_{q-p+2, a}^{q-p+2}+\int_{-R_{3}}^{R_{3}} \max _{|x| \leq C_{2} C_{12}}|W(t, x)| d t<+\infty .
$$

This contradicts the fact that $\left\{d_{n}\right\}_{n=1}^{\infty}$ is unbounded, and so $\left\{\left\|u_{n}\right\|\right\}$ is unbounded. The proof is complete.

Proof of Theorem 1.4 In view of the proofs of Theorem 1.2 and Theorem 1.3, the conclusion of Theorem 1.4 holds. The proof is complete.

\section{Examples}

Example 4.1 Consider the following system:

$$
\frac{d}{d t}(|\dot{u}(t)| \dot{u}(t))-a(t)|u(t)|^{3} u(t)+\nabla W(t, u(t))=0, \quad \text { a.e. } t \in \mathbb{R},
$$


where $p=3, q=6, t \in \mathbb{R}, u \in \mathbb{R}^{N}, a \in C(\mathbb{R},(0, \infty))$ and $a$ satisfies (A)'. Let

$$
W(t, x)=a(t)\left(\sum_{i=1}^{m} a_{i}|x|^{\mu_{i}}-\sum_{j=1}^{n} b_{j}|x|^{e_{j}}\right)
$$

where $\mu_{1}>\mu_{2}>\cdots>\mu_{m}>\varrho_{1}>\varrho_{2}>\cdots>\varrho_{j}>5, a_{i}, b_{j}>0, i=1, \ldots, m, j=1, \ldots, n$. Let

$$
W_{1}(t, x)=a(t) \sum_{i=1}^{m} a_{i}|x|^{\mu_{i}}, \quad W_{2}(t, x)=a(t) \sum_{j=1}^{n} b_{j}|x|^{\varrho_{j}} .
$$

Then it is easy to check that all the conditions of Theorem 1.3 are satisfied with $\mu=\mu_{m}$ and $\varrho=\varrho_{1}$. Hence, problem (4.1) has an unbounded sequence of homoclinic solutions.

Example 4.2 Consider the following system:

$$
\frac{d}{d t}\left(|\dot{u}(t)|^{-1 / 2} \dot{u}(t)\right)-a(t)|u(t)| u(t)+\nabla W(t, u(t))=0, \quad \text { a.e. } t \in \mathbb{R},
$$

where $p=3 / 2, q=5 / 2, t \in \mathbb{R}, u \in \mathbb{R}^{N}, a \in C(\mathbb{R},(0, \infty))$ and $a$ satisfies (A)'. Let

$$
W(t, x)=a(t)\left[a_{1}|x|^{\mu_{1}}+a_{2}|x|^{\mu_{2}}-b_{1}(\cos t)|x|^{\varrho_{1}}-b_{2}|x|^{\varrho_{2}}\right]
$$

where $\mu_{1}>\mu_{2}>\varrho_{1}>\varrho_{2}>3, a_{1}, a_{2}>0, b_{1}, b_{2}>0$. Let

$$
W_{1}(t, x)=a(t)\left(a_{1}|x|^{\mu_{1}}+a_{2}|x|^{\mu_{2}}\right), \quad W_{2}(t, x)=a(t)\left[b_{1}(\cos t)|x|^{\varrho_{1}}+b_{2}|x|^{\varrho_{2}}\right] .
$$

Then it is easy to check that all the conditions of Theorem 1.4 are satisfied with $\mu=\mu_{2}$ and $\varrho=\varrho_{1}$. Hence, by Theorem 1.4, problem (4.2) has an unbounded sequence of homoclinic solutions.

\section{Competing interests}

The authors declare that they have no competing interests.

\section{Authors' contributions}

All authors read and approved the final manuscript.

\section{Author details}

'College of Science, Guilin University of Technology, Guilin, Guangxi 541004, P.R. China. ${ }^{2}$ College of Science, Hunan University of Technology, Zhuzhou, Hunan 412000, P.R. China.

\section{Acknowledgements}

XS and QZ are supported by the Scientific Research Foundation of Guangxi Education Office (No. 201203YB093), Guangxi Natural Science Foundation (Nos. 2013GXNSFBA019004 and 2012GXNSFBA053013) and the Scientific Research Foundation of Guilin University of Technology. QMZ is supported by the NNSF of China (No. 11201138) and the Scientific Research Fund of Hunan Provincial Education Department (No. 12B034).

Received: 5 December 2012 Accepted: 7 May 2013 Published: 24 May 2013

\section{References}

1. Poincaré, H: Les méthodes nouvelles de la mécanique céleste. Gauthier-Villars, Paris (1897-1899)

2. Alves, $\mathrm{CO}$, Carriao, $\mathrm{PC}$, Miyagaki, $\mathrm{OH}$ : Existence of homoclinic orbits for asymptotically periodic systems involving Duffing-like equation. Appl. Math. Lett. 16(5), 639-642 (2003)

3. Carriao, PC, Miyagaki, OH: Existence of homoclinic solutions for a class of time-dependent Hamiltonian systems J. Math. Anal. Appl. 230(1), 157-172 (1999) 
4. Coti, ZV, Rabinowitz, PH: Homoclinic orbits for second-order Hamiltonian systems possessing superquadratic potentials. J. Am. Math. Soc. 4(4), 693-727 (1991)

5. Chen, CN, Tzeng, SY: Existence and multiplicity results for homoclinic orbits of Hamiltonian systems. Electron. J. Differ. Equ. 1997, 7 (1997)

6. Ding, YH: Existence and multiplicity results for homoclinic solutions to a class of Hamiltonian systems. Nonlinear Anal. 25(11), 1095-1113 (1995)

7. Izydorek, M, Janczewska, J: Homoclinic solutions for a class of the second-order Hamiltonian systems. J. Differ. Equ. 219(2), 375-389 (2005)

8. Korman, P, Lazer, AC: Homoclinic orbits for a class of symmetric Hamiltonian systems. Electron. J. Differ. Equ. 1994, 1 (1994)

9. Korman, P, Lazer, AC, Li, Y: On homoclinic and heteroclinic orbits for Hamiltonian systems. Differ. Integral Equ. 10(2), 357-368 (1997)

10. Lu, YF, Li, CY, Zhong, SZ, Zhang, WJ: Homoclinic orbits for a class of Hamiltonian systems with potentials changing sign. Ann. Differ. Equ. 21(3), 370-372 (2005)

11. Lv, X, Lu, SP, Yan, P: Existence of homoclinic solutions for a class of second-order Hamiltonian systems. Nonlinear Anal. 72(1), 390-398 (2010)

12. Omana, W, Willem, M: Homoclinic orbits for a class of Hamiltonian systems. Differ. Integral Equ. 5(5), 1115-1120 (1992)

13. Rabinowitz, PH: Homoclinic orbits for a class of Hamiltonian systems. Proc. R. Soc. Edinb. A 114(1-2), 33-38 (1990)

14. Rabinowitz, PH, Tanaka, K: Some results on connecting orbits for a class of Hamiltonian systems. Math. Z. 206(3), 473-499 (1991)

15. Tang, XH, Xiao, L: Homoclinic solutions for a class of second-order Hamiltonian systems. Nonlinear Anal. 71(3-4), 1140-1152 (2009)

16. Tang, XH, Xiao, L: Homoclinic solutions for nonautonomous second-order Hamiltonian systems with a coercive potential. J. Math. Anal. Appl. 351(2), 586-594 (2009)

17. Tang, $\mathrm{XH}, \mathrm{XiaO}, \mathrm{L}$ : Homoclinic solutions for ordinary $p$-Laplacian systems with a coercive potential. Nonlinear Anal. 71(3-4), 1124-1132 (2009)

18. Tang, $X H$, Lin, $X Y$ : Existence of infinitely many homoclinic orbits in Hamiltonian systems. Proc. R. Soc. Edinb. A 141, 1103-1119 (2011)

19. Zhang, QF, Tang, XH: Existence of homoclinic solutions for a class of asymptotically quadratic non-autonomous Hamiltonian systems. Math. Nachr. 285(5-6), 778-789 (2012)

20. Zhang, $X Y$, Tang, $X H$ : A note on the minimal periodic solutions of nonconvex superlinear Hamiltonian system. Appl. Math. Comput. 219, 7586-7590 (2013)

21. Chen, $\mathrm{P}, \mathrm{Tang}, \mathrm{XH}$ : New existence of homoclinic orbits for a second-order Hamiltonian system. Comput. Math. Appl. 62(1), 131-141 (2011)

22. Salvatore, A: On the existence of homoclinic orbits for a second-order Hamiltonian system. Differ. Integral Equ. 10(2), 381-392 (1997)

23. Salvatore, A: Homoclinic orbits for a class of strictly convex Hamiltonian systems. Dyn. Syst. Appl. 6, 153-164 (1997)

24. Benci, V, Fortunato, D: Weighted Sobolev space and the nonlinear Dirichlet problem in unbounded domains. Ann. Mat. Pura Appl. 121, 319-336 (1979)

25. Royden, HL: Real Analysis, 2nd edn. Macmillan Co., New York (1968)

26. Rabinowitz, PH: Minimax Methods in Critical Point Theory with Applications to Differential Equations. CBMS Regional Conf. Ser. in Math., vol. 65. Am. Math. Soc., Providence (1986)

27. Adams, RA, Fournier, JJF: Sobolev Spaces, 2nd edn. Academic Press, Amsterdam (2003)

doi:10.1186/1687-2770-2013-137

Cite this article as: Shi et al.: Existence of homoclinic orbits for a class of $p$-Laplacian systems in a weighted Sobolev space. Boundary Value Problems 2013 2013:137.

\section{Submit your manuscript to a SpringerOpen ${ }^{\circ}$ journal and benefit from:}

- Convenient online submission

Rigorous peer review

- Immediate publication on acceptance

- Open access: articles freely available online

- High visibility within the field

- Retaining the copyright to your article 